\title{
Post Biological Assessment of Faunal Resources in The Samar Island Natural Park.
}

\author{
Teofanes A. Patindol \\ Department of Forest Science, College of Forestry and Environmental Science, \\ Visayas State University, Baybay City, Leyte, Philippines
}

\section{ABSTRACT}

Samar Island Natural Park (SINP) is regarded as one of the most important areas in terms of biodiversity in the island of Samar and within the greater Mindanao faunal region. Five priority watersheds within the SINP were surveyed for the post biological assessment. These were the watersheds of Taft, Can-avid, Basey, Suribao and Catubig. Standard faunal survey methods such as transect survey, mist netting, and trapping were used during the entire duration of the study. The methods were augmented with ocular observations and ethnobiological interviews.

Of the 182 species of terrestrial vertebrates recorded from SINP, 121 species were birds, 20 were mammals, 18 amphibians and 23 reptiles. The overall endemism is $40 \%$. The 121 species of birds belong to 15 orders and 45 families. Of these, $26 \%$ are endemic to the Philippines and $12 \%$ are endemic to the greater Mindanao faunal region. Among avian species, thirteen (11\%) are considered threatened or at risk.

of the 11 mammals, which belong to 6 orders and 9 families, $45 \%$ are endemic to the country and $15 \%$ are endemic to the greater Mindanao faunal region. Seven (35\%) of these species are considered threatened or at risk.

Of the 18 species of amphibians recorded, 2 are introduced and the rest are native to the Philippines. Of the 23 reptiles, $39 \%$ are endemic to the country and $3 \%$ are endemic to the greater Mindanao faunal region. Among herpetofauna, only one species is considered threatened or at risk.

Samar holds high diversity and high endemism and contains a remarkable number of threatened species being recognized by the international arena in biological diversity conservation, most of which have strong affinity to the Mindanao faunal region. The existing data are sufficient to further highlight the need to establish SINP as conservation priority site in the region.

Keywords: Samar Island Natural Park, faunal diversity, endemism, threatened species

Correspondence: T. A. Patindol Address: Department of Forest Science, College of Forestry and Environmental Science, Visayas State University, Baybay City, Leyte, Philippines Tel No.: (63-053) 5637552 E-mail: teofanes.patindol@vsu.edu.ph DOI: $10.32945 /$ atr3824.2016 


\section{INTRODUCTION}

The Samar Island Biodiversity Project (SIBP) was implemented by the United Nations Development Program (UNDP) and the Global Environmental Facility (GEF) through the Department of Environment and Natural Resources (DENR) (UNDP-GEF-DENR, 2007). The project aimed to promote biodiversity conservation and sustainable management of forest resources in Samar Island through a community-based conservation regime. Samar Island has been selected to form part of the GEF by having the largest forested area in Region 8. Although most parts of forest in Samar Island had been logged in the last few decades, its biological resources could have been recovering already through natural regeneration.

The upgrading of the conservation status of the covered area into a natural park, to become the Samar Island Natural Park (SINP), made it imperative to have a more precise accounting of the biological resources therein. Baseline data on the current state of biological resources are basic requisites for planning and decision making for current and future management programs. Baseline data provide insights on the current ecological health status of forest resources, which is also the basis of whether such resources are valuable enough to justify investments in conservation. Baseline data are also providing benchmark with which to compare future biological resource assessment when the interest is to check whether such resources are improving or suffering from degradation. Benchmark also helps in determining the seriousness of the need for action in the conservation of biodiversity in the park.

A biological resources assessment (BRA) in the SINP area has been conducted in 2002 to 2003 as one major activity of the SIBP (SEAMEOSEARCA, 2004). The BRA was conducted to establish baseline information on biodiversity, conservation values, status of critical natural habitats and to validate SINP's indicative management zones. The BRA in 2002-03 was undertaken by a group of experts in the fields of taxonomy and botany. Their output was a product of a thorough field methodology for an exhaustive enumeration of floral and faunal species along transects in 8 selected watersheds. It produced a very valuable document on biological diversity of the sites and has ultimately confirmed the worth of protecting the natural forests in Samar Island. For the national and international level of interests in line with biodiversity conservation, it represents a big stride in SINP's development programs.

The general objective of the post biological resource assessment was to revalidate the previous baseline information on existing flora and fauna resources, including critical habitats and indicators identified and conduct an analysis of changes and trends for effective management of SINP. Specifically the study aimed to identify patterns of fauna-habitat associations that will provide guidance for the design and implementation of management interventions for particular species' survival within SINP and across their range and to provide bases for policy recommendation 
and inputs in the updating of the SINP management plan.

This article presents the result of the post biological assessment of the faunal resources of SINP which include the four major groups of vertebrates, namely: birds, mammals, reptiles and amphibians.

\section{METHODOLOGY}

\section{The Study Area}

The Samar Island Natural Park as provided for by Presidential Decree (PD) 442 covers 333,330 hectares of protected area and buffer zone of 124,500 hectares. It has 8 watersheds: Suribao, Can-avid, Catubig, Taft, Basey, Dolores, Gandara and Pambujan. This study has covered the first five watersheds in the assessment.

The SINP has abundant rainfall with mean annual rainfall of about $3,600 \mathrm{~mm}$. Climatic types II and IV prevail in the area. Type II is characterized by having no dry season, with pronounced maximum rainfall period in the months of December and January. Type IV has more or less even distribution of rainfall throughout the year, or without period with maximum rains. Within the protected area, only about $2 \%$ can be considered flat, $14 \%$ as undulating to flat and the rest is rolling to moderately steep and very steeply mountainous. The soil is clay loam to clay.

The geology is mostly Miocene to Holocene sedimentary rocks. Where the SINP is located, the sedimentary formation generally consists of basement rocks, sometimes with overlying clastic rocks or limestone. The area consists of an interior highland with marked accordant peaks and a surrounding limestone or karst terrain. The southern peninsula is made up of jungle-covered limestone ridge that appears to be a younger coral reef. The central highlands are principally of igneous complex intercalated with metamorphosed sedimentary rocks. With the presence of thick mantle of laterite soil, indicates that the igneous rocks have been subjected to intense mechanical and chemical weathering. A more complete physical description of the SINP area is presented in the SINP Management Plan 2006 to 2016 (UNDP-GEF-DENR, 2007).

\section{FaunalSurvey}

Faunal survey was conducted to cover all four major vertebrate groups (reptiles, amphibians, birds, and mammals). Different techniques or methods of faunal inventory include trapping of non-volant mammals, mist netting of non-volant mammals, and transect survey of birds, reptiles and amphibians. Indirect methods of faunal survey such as those using footprints, fecal droppings, roosting and nesting sites, other physical evidences were likewise used. Ethnobiological interviews that may indicate the probable presence of wildlife in the area were also employed. 
Avifaunal Survey

Composition and distribution of avian species were assessed using the transect survey method based on Mallari (1992). A transect line measuring 2 kilometers was set in every sampling site. All species of birds encountered along the transect were recorded. For each species seen or heard, the following information were noted: species name, number of individuals, and habitat types. The avifaunal inventory was conducted early in the morning (from 6:00 to 9:00) and late in the afternoon (from 3:00 to 6:00). Identification was made down to the species level whenever possible. Nomenclature and classification were based on Kennedy et al. (2000).

\section{Mammalian Survey}

Mist nets for volant mammals and live traps for non-volant mammals were used in the mammalian survey. Nomenclature and classification were based on Rabor (1986).

Mist-netting stations were set up in strategic locations either singly or a series and operated for days in specific study sites. Each net has an average mesh size of $36 \mathrm{~mm}$ and an average height of 2 meters. Nets were set 2 meter high with a ground clearance of about 0.5 meter. Species captured were identified up to the species level whenever possible.

Trapping of non-volant mammals involved setting of cage traps baited with cooked coconut meat, sliced ripe mango or dried fish, on the ground near fallen logs or holes, along possible runways or root system of trees or stumps. Similarly, trapped or captured animals were identified up to the species level whenever possible. Tracks and fecal matters were also used to estimate the presence of wildlife in each transect route used in avifaunal survey.

Herpetofaunal Survey

Random sampling of reptiles and amphibians was done whenever possible along the transect route used for the avifaunal survey. Sampling was done along streams, rivers or near bodies of water or any site believed to be harborages of herpetofauna. Animals were either collected by hand or captured by nets for further verification. Nomenclature and classification was based on Alcala (1986) and Alcala and Brown (1998).

\section{Other Methods of Faunal Survey}

Ethnobiological interviews were conducted to determine the presence of other vertebrates that were not recorded during the field observations. Information on vernacular or local names, habitat type, socioeconomic importance and other patterns of behavior were noted. Other activities 
related to the overall decline or loss of wildlife species in the area were also recorded.

\section{Data Presentation and Analysis}

Data recorded from the 5 watersheds were presented in tabular form showing the species composition to include order, family, scientific name, common name, and residency status. Conservation status of the different fauna was based on IUNC Rest list of Endangered Species (IUCN, 2015) and DENR DAO 2004-15 (DENR, 2004). Ecological role of each species and economic value of key species and threats of endangered species were also presented. Distribution of species was presented in tabular form.

Quantitative analysis of the data recorded from the 5 study sites was done using level of diversity and similarity. Ecological parameters such as index for species diversity using the Shannon-Weiner function $\left(\mathrm{H}^{\prime}\right)$ were computed for each site. Species assemblages of the 5 sites were compared using the Jaccard Index of Similarity.

Species Diversity

Diversity was computed for each sampling site using Shannon's Diversity Index (Magurran, 1988):

$$
\mathrm{H}^{\prime}=-\sum\left[\frac{\mathrm{n} 1}{\mathrm{n}}\right]\left[\ln \frac{\mathrm{n} 1}{\mathrm{n}}\right]
$$

where: $\quad H^{\prime}=$ the Shannon index of diversity

$\mathrm{n}_{1}=$ number of individuals per species and

$\mathrm{n}=$ total number of individuals

\section{Similarity Index}

The degree of similarity in species composition among sites was presented using a table on Jaccard indices. Jaccard index was calculated using the formula below:

$$
J=\frac{c}{a+b-c}
$$

where: $\mathrm{J}=$ the Jaccard index $\mathrm{a}=$ the number of species in the first population; $\mathrm{b}=$ the number of species in the second population; $\mathrm{c}=$ the number of species occurring in both populations. 


\author{
RESULTS AND DISCUSSION
}

AVIFAUNA

\title{
Species Composition
}

A total of 121 bird species were recorded in the five study sites (Table 1). These species belong to 15 orders and 45 families. The most represented order was Passeriformes with 60 species belonging to 23 families. The second most represented order was Columbiformes with 13 species. This was followed by Coraciiformes and Gruiformes with 10 and 6 species, respectively.

Table 1. List of birds recorded from five priority watersheds in Samar Island Natural Park, c.

\begin{tabular}{|c|c|c|c|c|}
\hline Order & Family Name & Scientific Name & Common Name & Status* \\
\hline Anseriformes & Anatidae & Anas luzonica & Philippine mallard & Philippine Endemic \\
\hline Anseriformes & Anatidae & Dendrocygna arcuata & Wandering whistling duck & Resident \\
\hline Apodiformes & Apodidae & Collocalia mearnsi & Philippine swiftlet & Philippine Endemic \\
\hline Apodiformes & Apodidae & Collocalia troglodytes & Pygmy swiftlet & Philippine Endemic \\
\hline Apodiformes & Apodidae & Collocalia esculenta & Glossy swiftlet & Resident \\
\hline Caprimulgiformes & Podargidae & Batrachostomus septimus & Philippine frogmouth & Philippine Endemic \\
\hline Caprimulgiformes & Caprimulgidae & Caprimulgus affinis & Savannah nightjar & Resident \\
\hline Caprimulgiformes & Caprimulgidae & Caprimulgus manillensis & Philippine nightjar & Resident \\
\hline Charadriformes & Scolopacidae & Actitis bypoleucos & Common sandpiper & Migrant \\
\hline Charadriformes & Charadriidae & Chardrius leschenaultii & Greater sand-plover & Migrant \\
\hline Ciconiiformes & Ardeidae & Egretta garzetta & Little egret & Migrant \\
\hline Ciconiiformes & Ardeidae & Bubulcus ibis & Cattle egret & Resident \\
\hline Ciconiiformes & Ardeidae & Ixobrycbus cinnamomeus & Cinnamon bittern & Resident \\
\hline Ciconiiformes & Ardeidae & Nycticorax caledonicus & Rufous night heron & Resident \\
\hline Columbiformes & Columbidae & Chalcophaps indica & Common emerald dove & Resident \\
\hline Columbiformes & Columbidae & Columba vitiensis & Metallic pigeon & Resident \\
\hline Columbiformes & Columbidae & Ducula aenea & Green imperial pigeon & Resident \\
\hline Columbiformes & Columbidae & Gallicolumba criniger & Mindanao bleeding heart & Mindanao Endemic \\
\hline Columbiformes & Columbidae & Geopelia striata & Zebra dove & Resident \\
\hline Columbiformes & Columbidae & Macropygia phasianella & Reddish cuckoo dove & Resident \\
\hline Columbiformes & Columbidae & Phapitreron ametbystina & Amethyst brown dove & Philippine Endemic \\
\hline Columbiformes & Columbidae & Phapitreron leucotis & White-eared brown fruit dove & Philippine Endemic \\
\hline Columbiformes & Columbidae & Ptilinopus lechancher & Black chinned fruit dove & Resident \\
\hline Columbiformes & Columbidae & Ptilinopus occipitalis & Yellow-breasted fruit dove & Philippine Endemic \\
\hline Columbiformes & Columbidae & Streptopelia chinensis & Spotted dove & Resident \\
\hline Columbiformes & Columbidae & Treron pompadora & Pompadour green pigeon & Philippine Endemic \\
\hline Columbiformes & Columbidae & Treron vernans & Pink-necked green pigeon & Resident \\
\hline Coraciiformes & Alcedinidae & Alcedo argentatus & Silvery king fisher & Mindanao Endemic \\
\hline Coraciiformes & Alcedinidae & Ceyx lepidus & variable dwarf-kingfisher & Philippine Endemic \\
\hline Coraciiformes & Alcedinidae & Ceyx melanurus & Philippine dwarf-kingfisher & Philippine Endemic \\
\hline Coraciiformes & Alcedinidae & Halcyon cbloris & White-collard king fisher & Resident \\
\hline Coraciiformes & Alcedinidae & Halcyon coromanda & Rudy kingfisher & Philippine Endemic \\
\hline Coraciiformes & Alcedinidae & Halcyon smymensis & White throated king fisher & Resident \\
\hline Coraciiformes & Bucerotidae & Buceros bydrocorax & Rofous hornbill & Philippine Endemic \\
\hline Coraciiformes & Bucerotidae & Penelopides affinis & Tarictic hornbill & Mindanao Endemic \\
\hline Coraciiformes & Coraciidae & Eurystomus orientalis & Dollard bird & Resident \\
\hline Coraciiformes & Meropidae & Merops viridis & Blue-throated bee-eater & Resident \\
\hline Cuculiformes & Cuculidae & Cacomantis variolosus & Brush cuckoo & Resident \\
\hline Cuculiformes & Cuculidae & Centropus melanops & Mountain coucal & Mindanao Endemic \\
\hline Cuculiformes & Cuculidae & Centropus viridis & Philippine coucal & Philippine Endemic \\
\hline Cuculiformes & Cuculidae & Eudynamys scolopacea & Common koel & Resident \\
\hline Cuculiformes & Cuculidae & Phaenicphaeus curvirostris & Chestnut -breasted malkoha & Resident \\
\hline Falconiformes & Accipitridae & Haliastur indus & Brahminy kite & Resident \\
\hline Falconiformes & Accipitridae & Pithecophaga jefferyi & Philippine Eagle & Philippine Endemic \\
\hline Falconiformes & Accipitridae & Spilornis cheela & Crested serpent eagle & Resident \\
\hline Falconiformes & Accipitridae & Spizaetus philippensis & Philippine Hawk-eagle & Philippine Endemic \\
\hline Falconiformes & Falconidae & Microbierax erythrogenys & Philippine falconet & Philippine Endemic \\
\hline
\end{tabular}


Table 1.Continuation

\begin{tabular}{|c|c|c|c|c|}
\hline Order & Family Name & Scientific Name & Common Name & Status* \\
\hline Galliformes & Megapodiidae & Megapodius cumingii & Tabon scrubfowl & Resident \\
\hline Gruiformes & Rallidae & Amanromis phoenicurus & White-breasted waterhen & Resident \\
\hline Gruiformes & Rallidae & Gallinula chloropus & Common moorhen & Resident \\
\hline Gruiformes & Rallidae & Galliralus torquatus & Barred rail & Resident \\
\hline Gruiformes & Rallidae & Porzana cinerea & White browed crake & Resident \\
\hline Gruiformes & Rallidae & Rallina eurizonoides & Slaty-legged rake & Resident \\
\hline Gruiformes & Turnicidae & Turnix suscitator & Barred button quail & Resident \\
\hline Passeriformes & Artamindae & Artamus leucorbynchus & White-breasted wood swallow & Resident \\
\hline Passeriformes & Campephagidae & Lalage nigra & Pied triller & Resident \\
\hline Passeriformes & Corvidae & Corvus macrorbynchus & Large-billed crow & Resident \\
\hline Passeriformes & Dicaeidae & Dicaeum anthonyi & Flame-crowned flower pecker & Philippine Endemic \\
\hline Passeriformes & Dicaeidae & Dicaeum bypoleucum & Buzzing flower pecker & Philippine Endemic \\
\hline Passeriformes & Dicaeidae & Dicaeum nigrilore & Olive-capped flowerpecker & Philippine Endemic \\
\hline Passeriformes & Dicaeidae & Dicaeum trogonostigma & Orange-bellied flowerpecker & Resident \\
\hline Passeriformes & Dicruridae & Dicrurus balicassius & Balicassiao & Philippine Endemic \\
\hline Passeriformes & Dicruridae & Dicrurus bottentottus & Spangled drongo & Resident \\
\hline Passeriformes & Estrildidae & Erythrura coloria & Red-eared Parrot-finch & Mindanao Endemic \\
\hline Passeriformes & Estrildidae & Lonchura leucogastra & White bellied munia & Resident \\
\hline Passeriformes & Estrildidae & Lonchura malacca & Chestnut munia & Resident \\
\hline Passeriformes & Estrildidae & Lonchura punctulata & Scaly-breasted munia & Resident \\
\hline Passeriformes & Estrildidae & Padda oryzivora & Java sparrow & Resident \\
\hline Passeriformes & Eurylaimidae & Eurylaimus steerii & Wattled broadbill & Mindanao Endemic \\
\hline Passeriformes & Fringillidae & Pyrrbula leucogenis & White-cheeked bullfinch & Philippine Endemic \\
\hline Passeriformes & Laniidae & Lanius cristatus & Brown shrike & Migrant \\
\hline Passeriformes & Laniidae & Lanius schach & Long tailed shrike & Resident \\
\hline Passeriformes & Motacillidae & Anthus novaeseelandiae & Richard's pipit & Resident \\
\hline Passeriformes & Motacillidae & Motacilla cinerea & Grey wagtail & Migrant \\
\hline Passeriformes & Motacillidae & Motacilla flava & Yellow wagtail & Migrant \\
\hline Passeriformes & Muscicapidae & Brachypteryx montana & White browed shortwing & Resident \\
\hline Passeriformes & Muscicapidae & Eumyias panayensis & Mountain verideter flycatcher & Resident \\
\hline Passeriformes & Muscicapidae & Ficedula crypta & Cryptic flycatcher & Mindanao Endemic \\
\hline Passeriformes & Muscicapidae & Rhipidura javanica & Pied fantail & Resident \\
\hline Passeriformes & Muscicapidae & Rhipidura superciliaris & Blue fantail & Mindanao Endemic \\
\hline Passeriformes & Muscicapidae & Terpsiphone cinnamomea & Rofous paradise-flying catcher & Resident \\
\hline Passeriformes & Nectariniidae & Aethopyga pulcherrina & Metallic-winged sunbird & Philippine Endemic \\
\hline Passeriformes & Nectariniidae & Nectarinia jugularis & Olive-backed sunbird & Resident \\
\hline Passeriformes & Nectariniidae & Nectarinia sperata & purple throated sunbird & Resident \\
\hline Passeriformes & Oriolidae & Irena puella & Philippine fairy blue bird & Philippine Endemic \\
\hline Passeriformes & Oriolidae & Oriolus chinensis & Black naped oriole & Resident \\
\hline Passeriformes & Pachycephalidae & Pachycephala bomeyeri & White-vented whistler & Near Endemic \\
\hline Passeriformes & Pachycephalidae & Pachycephala pbilippinensis & Yellow-bellied whistler & Philippine Endemic \\
\hline Passeriformes & Phasianidae & Coturnix chinensis & Blue breasted quail & Resident \\
\hline Passeriformes & Phasianidae & Gallus gallus & Red jungle fowl & Resident \\
\hline Passeriformes & Pittadae & Pitta kochi & Whiskered pitta & Resident \\
\hline Passeriformes & Pittadae & Pitta steerii & Steere's pitta & Mindanao Endemic \\
\hline Passeriformes & Ploceidae & Passer mantanus & Eurasian tree sparrow & Resident \\
\hline Passeriformes & Pycnonotidae & Hypsipetes everetti & Yellowish bulbul & Mindanao Endemic \\
\hline Passeriformes & Pycnonotidae & Hypsipetes philippinus & Philippine bulbul & Philippine Endemic \\
\hline Passeriformes & Pycnonotidae & Pycnonotus goiaver & Yellow vented bulbul & Resident \\
\hline Passeriformes & Pycnonotidae & Pycnonotus urostictus & Yellow-wattled bulbul & Philippine Endemic \\
\hline Passeriformes & Strigidae & Bubo philippensis & Philippine eagle-owl & Philippine Endemic \\
\hline Passeriformes & Strigidae & Ninox sculata & Brown hawk owl & Resident \\
\hline Passeriformes & Sturnidae & Aplonis panayensis & Asian glossy starling & Resident \\
\hline Passeriformes & Sturnidae & Sarcops calvus & Coleto & Near Endemic \\
\hline Passeriformes & Sylviidae & Cisticola juncidis & Zitting cisticola & Resident \\
\hline Passeriformes & Sylviidae & Megalurus palustris & Striated grassbird & Resident \\
\hline Passeriformes & Sylviidae & Megalurus timoriensis & Tawny grassbird & Resident \\
\hline Passeriformes & Sylviidae & Orthotomus castaneiceps & Philippine tailorbird & Philippine Endemic \\
\hline Passeriformes & Sylviidae & Orthotomus samarensis & Yellow-breasted tailorbird & Mindanao Endemic \\
\hline Passeriformes & Sylviidae & Phylloscopus borealis & Arctic warbler & Migrant \\
\hline Passeriformes & Sylviidae & Phylloscopus cebuensis & Lemon-throated leaf warbler & Philippine Endemic \\
\hline Passeriformes & Sylviidae & Phylloscopus trivirgatus & Mountain leaf warbler & Resident \\
\hline Passeriformes & Timaliidae & Macronous striaticeps & Brown tit babbler & Mindanao Endemic \\
\hline Passeriformes & Timaliidae & Micromacronous leytensis & Miniature tit babbler & Mindanao Endemic \\
\hline Passeriformes & Timaliidae & Ptilocicula mindanensis & Streaked ground babbler & Mindanao Endemic \\
\hline Passeriformes & Turdidae & Zoothera dauma & Scaly ground thrush & Migrant \\
\hline Passeriformes & Zosteropidae & Zosterops montanus & Mountain white eye & Resident \\
\hline
\end{tabular}

Source: Kennedy, et al. 2000. 
Columbidae was the most represented family with 8 species of dove and 5 species of pigeon. It was followed by family Sylviidae with 8 species. Most Columbidae species are frugivoruos and are often encountered in brushlands bordering secondary forest areas. Their ability as good agents for seed dispersal enables them to play vital role in the forest ecosystem. On the other hand, members of the family Sylviidae are generally insectivorous, thus effective to control insect populations. This was followed by three families represented by 6 species each: Dicaeidae, Alcedinidae, and Muscicapidae. Another three families were represented by 5 species each: Cuculidae, Rallidae and Estrildidae. This indicates that the avifauna of the watersheds on Samar Island was composed largely of frugivorous birds such as pigeons and doves; insectivorous birds such as flycatchers, warblers, cuckoos and coucals; seed eaters such as munias; predators such as the kingfishers and rails; and nectivores such as the flowerpeckers.

The five priority watersheds were also dominated by another three families having 4 species each, including family Accipitridae (raptors), Pycnonotidae (bulbuls) and Psittacidae (parrots), as well as two avian families represented with 3 species each, such as Apodidae (swifts) and Motacillidae (wagtails). This assemblage of species indicates that the avifauna of Samar was composed of large birds of prey, frugivorous and insectivorous birds such as sunbirds and parrots; as well as sweeping insectivorous such as swifts and swiftlets.

The result of the study shows that the lowland forests of Samar is home to diverse species of birds which may indicate the ecological conditions of various habitats and form an important link between the food web and nutrient cycle. The natural vegetation in the forests includes a wide range of trees of different species, sizes and heights, thus creates several canopy layers representing various ecological niches. Such niches are used by a diverse number of birds for foraging, nesting and roosting due to their heterogeneity of microhabitats and available rich food sources. For example, the emergent canopy layer is mainly occupied by warbler (Silvidae) species, whereas the ground layer is occupied by babbler (Timaliidae) species.

Of the 121 avian species recorded, forty-seven (39\%) were endemic to the Philippines, of which fourteen (12\%) were endemic to the Mindanao faunal region; three $(2 \%)$ were near endemic, and eight $(7 \%)$ were migrant. Several interesting similarity may be observed between the result of the present study and the 2003 BRA in terms of endemic species. But the present study has higher percent endemism owing to the slightly lower number of species recorded to the previous one. It is noteworthy that although the number of endemic species was the same, there was slight difference in composition of endemics between the two surveys.

Conservation status of avian species recorded in the five priority watersheds were assessed based on DENR DAO 2004-15. Some 13 species of birds were considered threatened. These include the critically 
endangered Pithecophaga jefferyi and endangered Gallicolumba criniger. The Philippine cockatoo (Cacatua haematuropygia), a critically endangered species which was recorded in the previous BRA was not observed during the present study. This species may be present in other watersheds within Samar Island being part of its geographic range. Some 10 species were vulnerable, such as Anas luzonica, Spizaetus philippensis, Bubo philippensis, Megapodius cimingii, Alcedo argentatus, Ceyx melanurus, Buceros hydrocorax, Eurylaimus steerii, Pitta steerii and Tanygnathus lucionensis.

\section{Bird Species Distribution, Diversity and Similarity}

The distribution of bird species in the five priority watersheds is shown in Table 2. Catubig had the highest number of species with a total of 101; Suribao had the lowest with 55. Taft, Can-avid and Basey had 66, 62 and 85 species, respectively. All sites had high species diversity with Catubig Watershed having the highest index of diversity of 3.92 and Taft Watershed having the lowest with 3.25.

Table 2. Distribution of bird species recorded from five priority watersheds in Samar Island Natural Park, c. 2011

\begin{tabular}{|c|c|c|c|c|c|c|}
\hline Scientific Name & Common Name & 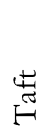 & 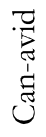 & 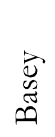 & 疋 & 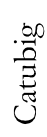 \\
\hline Alcedo argentatus & Silvery king fisher & $\mathrm{x}$ & $\mathrm{x}$ & $\mathrm{x}$ & $\mathrm{x}$ & $\mathrm{x}$ \\
\hline Artamus leucorbynchus & White-breasted wood swallow & $\mathrm{x}$ & $\mathrm{x}$ & $\mathrm{x}$ & $\mathrm{x}$ & $x$ \\
\hline Bubo philippensis & Philippine eagle-owl & $\mathrm{x}$ & $\mathrm{x}$ & $\mathrm{x}$ & $\mathrm{x}$ & $\mathrm{x}$ \\
\hline Bubulcus ibis & Cattle egret & $\mathrm{x}$ & $\mathrm{x}$ & $\mathrm{x}$ & $\mathrm{x}$ & $\mathrm{x}$ \\
\hline Buceros bydrocorax & Rofous hornbill & $\mathrm{x}$ & $\mathrm{x}$ & $\mathrm{x}$ & $\mathrm{x}$ & $\mathrm{x}$ \\
\hline Centropus melanops & Mountain coucal & $\mathrm{x}$ & $\mathrm{x}$ & $\mathrm{x}$ & $\mathrm{x}$ & $\mathrm{x}$ \\
\hline Chalcophaps indica & Common emerald dove & $\mathrm{x}$ & $\mathrm{x}$ & $\mathrm{x}$ & $\mathrm{x}$ & $\mathrm{x}$ \\
\hline Dicaeum bypoleucum & Buzzing flower pecker & $\mathrm{x}$ & $\mathrm{x}$ & $\mathrm{x}$ & $\mathrm{x}$ & $\mathrm{x}$ \\
\hline Dicrurus balicassius & Balicassiao & $\mathrm{x}$ & $\mathrm{x}$ & $\mathrm{x}$ & $\mathrm{x}$ & $\mathrm{x}$ \\
\hline Dryocopus javensis & White bellied wood pecker & $\mathrm{x}$ & $\mathrm{x}$ & $\mathrm{x}$ & $\mathrm{x}$ & $\mathrm{x}$ \\
\hline Eurystomus orientalis & Dollard bird & $\mathrm{x}$ & $\mathrm{x}$ & $\mathrm{x}$ & $x$ & $\mathrm{x}$ \\
\hline Ficedula crypta & Cryptic flycatcher & $\mathrm{x}$ & $\mathrm{x}$ & $\mathrm{x}$ & $\mathrm{x}$ & $\mathrm{x}$ \\
\hline Halcyon smyrnensis & White throated king fisher & $\mathrm{x}$ & $\mathrm{x}$ & $\mathrm{x}$ & $\mathrm{x}$ & $\mathrm{x}$ \\
\hline Haliastur indus & Brahminy kite & $\mathrm{x}$ & $\mathrm{x}$ & $\mathrm{x}$ & $x$ & $\mathrm{x}$ \\
\hline Hypsipetes everetti & Yellowish bulbul & $\mathrm{x}$ & $\mathrm{x}$ & $\mathrm{x}$ & $\mathrm{x}$ & $\mathrm{x}$ \\
\hline Hypsipetes philippinus & Philippine bulbul & $\mathrm{x}$ & $\mathrm{x}$ & $\mathrm{x}$ & $\mathrm{x}$ & $\mathrm{x}$ \\
\hline Irena puella & Philippine fairy blue bird & $\mathrm{x}$ & $\mathrm{x}$ & $\mathrm{x}$ & $\mathrm{x}$ & $\mathrm{x}$ \\
\hline Lanius cristatus & Brown shrike & $\mathrm{x}$ & $\mathrm{x}$ & $\mathrm{x}$ & $\mathrm{x}$ & $\mathrm{x}$ \\
\hline Lonchura malacca & Chestnut munia & $\mathrm{x}$ & $\mathrm{x}$ & $\mathrm{x}$ & $\mathrm{x}$ & $\mathrm{x}$ \\
\hline Loriculus philippensis & Colasisi & $\mathrm{x}$ & $\mathrm{x}$ & $\mathrm{x}$ & $x$ & $\mathrm{x}$ \\
\hline Motacilla cinerea & Grey wagtail & $\mathrm{x}$ & $\mathrm{x}$ & $\mathrm{x}$ & $\mathrm{x}$ & $\mathrm{x}$ \\
\hline Pachycephala bomeyeri & White-vented whistler & $\mathrm{x}$ & $\mathrm{x}$ & $\mathrm{x}$ & $\mathrm{x}$ & $\mathrm{x}$ \\
\hline Penelopides panini & Tarictic hornbill & $\mathrm{x}$ & $\mathrm{x}$ & $\mathrm{x}$ & $\mathrm{x}$ & $\mathrm{x}$ \\
\hline
\end{tabular}


Table 2. Continuation

\begin{tabular}{|c|c|c|c|c|c|c|}
\hline Scientific Name & Common Name & $\underset{ت}{\stackrel{\rightleftarrows}{E}}$ & 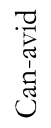 & 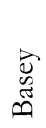 & 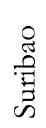 & 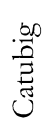 \\
\hline Phapitreron leucotis & White-eared brown fruit dove & $\mathrm{x}$ & $\mathrm{x}$ & $\mathrm{x}$ & $\mathrm{x}$ & $\mathrm{x}$ \\
\hline Pycnonotus goiaver & Yellow vented bulbul & $\mathrm{x}$ & $\mathrm{x}$ & $\mathrm{x}$ & $\mathrm{x}$ & $\mathrm{x}$ \\
\hline Rallina eurizonoides & Slaty-legged rake & $\mathrm{x}$ & $\mathrm{x}$ & $\mathrm{x}$ & $\mathrm{x}$ & $\mathrm{x}$ \\
\hline Sarcops calvus & Coleto & $\mathrm{x}$ & $\mathrm{x}$ & $\mathrm{x}$ & $\mathrm{x}$ & $\mathrm{x}$ \\
\hline Terpsiphone cinnamomea & Rofous paradise-flying catcher & $\mathrm{x}$ & $\mathrm{x}$ & $\mathrm{x}$ & $\mathrm{x}$ & $\mathrm{x}$ \\
\hline Treron pompadora & Pompadour green pigeon & $\mathrm{x}$ & $\mathrm{x}$ & $\mathrm{x}$ & $\mathrm{x}$ & $\mathrm{x}$ \\
\hline Treron vernans & Pink-necked green-pigeon & $\mathrm{x}$ & $\mathrm{x}$ & $\mathrm{x}$ & $\mathrm{x}$ & $\mathrm{x}$ \\
\hline Anas luzonica & Philippine mallard & $\mathrm{x}$ & $\mathrm{x}$ & $\mathrm{x}$ & & $\mathrm{x}$ \\
\hline Bolbopsittacus lunulatus & Guiabero & $\mathrm{x}$ & $\mathrm{x}$ & $\mathrm{x}$ & & $\mathrm{x}$ \\
\hline Ceyx melanurus & Philippine dwarf-kingfisher & $\mathrm{x}$ & $\mathrm{x}$ & $\mathrm{x}$ & & $\mathrm{x}$ \\
\hline Ninox sculata & Brown hawk owl & $\mathrm{x}$ & $\mathrm{x}$ & $\mathrm{x}$ & & $\mathrm{x}$ \\
\hline Orthotomus samarensis & Yellow-breasted tailorbird & $\mathrm{x}$ & $\mathrm{x}$ & $\mathrm{x}$ & & $\mathrm{x}$ \\
\hline Ptilocicula mindanensis & Streaked ground babbler & $\mathrm{x}$ & $\mathrm{x}$ & $\mathrm{x}$ & & $\mathrm{x}$ \\
\hline Spizaetus philippensis & Philippine Hawk-eagle & $\mathrm{x}$ & $\mathrm{x}$ & $\mathrm{x}$ & & $\mathrm{x}$ \\
\hline Streptopelia chinensis & Spotted dove & $\mathrm{x}$ & $\mathrm{x}$ & $\mathrm{x}$ & & $\mathrm{x}$ \\
\hline Batrachostomus septimus & Philippine frogmouth & $\mathrm{x}$ & $\mathrm{x}$ & & $\mathrm{x}$ & $\mathrm{x}$ \\
\hline Collocalia troglodytes & Pygmy swiftlet & $\mathrm{x}$ & $\mathrm{x}$ & & $\mathrm{x}$ & $\mathrm{x}$ \\
\hline Gallus gallus & Red jungle fowl & $\mathrm{x}$ & $\mathrm{x}$ & & $\mathrm{x}$ & $\mathrm{x}$ \\
\hline Megalaina haemacephala & Coppersmith barbet & $\mathrm{x}$ & $\mathrm{x}$ & & $\mathrm{x}$ & $\mathrm{x}$ \\
\hline Pitta steerii & Steere's pitta & $\mathrm{x}$ & $\mathrm{x}$ & & $\mathrm{x}$ & $\mathrm{x}$ \\
\hline Ptilinopus occipitalis & Yellow-breasted fruit dove & $\mathrm{x}$ & $\mathrm{x}$ & & $\mathrm{x}$ & $\mathrm{x}$ \\
\hline Amanrornis phoenicurus & White-breasted waterhen & $\mathrm{x}$ & & $\mathrm{x}$ & $\mathrm{x}$ & $\mathrm{x}$ \\
\hline Halcyon chloris & White-collard king fisher & $\mathrm{x}$ & & $\mathrm{x}$ & $\mathrm{x}$ & $\mathrm{x}$ \\
\hline Centropus viridis & Philippine coucal & & $\mathrm{x}$ & $\mathrm{x}$ & $\mathrm{x}$ & $\mathrm{x}$ \\
\hline Corvus macrorbynchus & Large-billed crow & & $\mathrm{x}$ & $\mathrm{x}$ & $\mathrm{x}$ & $\mathrm{x}$ \\
\hline Dicrurus hottentottus & Spangled drongo & & $\mathrm{x}$ & $\mathrm{x}$ & $\mathrm{x}$ & $\mathrm{x}$ \\
\hline Erythrura coloria & Red-eared Parrot-finch & & $\mathrm{x}$ & $\mathrm{x}$ & $\mathrm{x}$ & $\mathrm{x}$ \\
\hline Galliralus torquatus & Barred rail & & $\mathrm{x}$ & $\mathrm{x}$ & $\mathrm{x}$ & $\mathrm{x}$ \\
\hline Dicaeum nigrilore & Olive-capped flowerpecker & $\mathrm{x}$ & $\mathrm{x}$ & & & $\mathrm{x}$ \\
\hline Phylloscopus borealis & arctic warbler & $\mathrm{x}$ & $\mathrm{x}$ & & & $\mathrm{x}$ \\
\hline Actitis bypoleucos & Common sandpiper & $\mathrm{x}$ & $\mathrm{x}$ & $\mathrm{x}$ & & \\
\hline Caprimulgus affinis & Savannah nightjar & $\mathrm{x}$ & & $\mathrm{x}$ & & $\mathrm{x}$ \\
\hline Columba vitiensis & Metallic pigeon & $\mathrm{x}$ & & $\mathrm{x}$ & & $\mathrm{x}$ \\
\hline Gallicolumb a criniger & Mindanao bleeding heart & $\mathrm{x}$ & & $\mathrm{x}$ & & $\mathrm{x}$ \\
\hline Gallinula chloropus & Common moorhen & $\mathrm{x}$ & & $\mathrm{x}$ & & $\mathrm{x}$ \\
\hline Geopelia striata & Zebra dove & $\mathrm{x}$ & & $\mathrm{x}$ & & $\mathrm{x}$ \\
\hline Macropygia phasianella & Reddish cuckoo dove & $\mathrm{x}$ & & $\mathrm{x}$ & & $\mathrm{x}$ \\
\hline Oriolus chinensis & Black naped oriole & $\mathrm{x}$ & & $\mathrm{x}$ & & $\mathrm{x}$ \\
\hline Pithecophaga jefferyi & Philippine Eagle & $\mathrm{x}$ & & $\mathrm{x}$ & & $\mathrm{x}$ \\
\hline Prioniturus discurus & Blue-crowned racquet tail & $\mathrm{x}$ & & $\mathrm{x}$ & & $\mathrm{x}$ \\
\hline Phapitreron amethystina & Amethyst brown dove & $\mathrm{x}$ & & $\mathrm{x}$ & & $\mathrm{x}$ \\
\hline Merops viridis & Blue-throated bee-eater & $\mathrm{x}$ & & $\mathrm{x}$ & $\mathrm{x}$ & \\
\hline Collocalia mearnsi & Philippine swiftlet & & $\mathrm{x}$ & $\mathrm{x}$ & $\mathrm{x}$ & \\
\hline Nectarinia jugularis & Olive-backed sunbird & & $\mathrm{x}$ & & $\mathrm{x}$ & $\mathrm{x}$ \\
\hline Microhierax erythrogenys & Philippine falconet & & $\mathrm{x}$ & $\mathrm{x}$ & & $\mathrm{x}$ \\
\hline Nycticorax caledonicus & Rofous night heron & & $\mathrm{x}$ & $\mathrm{x}$ & & $\mathrm{x}$ \\
\hline Phylloscopus trivirgatus & Mountain leaf warbler & & $\mathrm{x}$ & $\mathrm{x}$ & & $\mathrm{x}$ \\
\hline Collocalia esculenta & Glossy swiftlet & & & $\mathrm{x}$ & $\mathrm{x}$ & $\mathrm{x}$ \\
\hline Hypsipetes palawanensis & Sulphur bellied bulbul & & & $\mathrm{x}$ & $\mathrm{x}$ & $\mathrm{x}$ \\
\hline
\end{tabular}


Table 2. Continuation

\begin{tabular}{|c|c|c|c|c|c|c|}
\hline Scientific Name & Common Name & 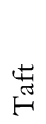 & 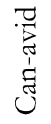 & 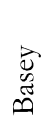 & 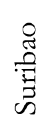 & $\frac{.00}{\frac{0}{2}}$ \\
\hline Lanius schach & Long tailed shrike & & & $\mathrm{x}$ & $\mathrm{x}$ & $\mathrm{x}$ \\
\hline Lonchura punctulata & Scaly-breasted munia & & & $\mathrm{x}$ & $\mathrm{x}$ & $\mathrm{x}$ \\
\hline Phylloscopus cebuensis & Lemon-throated leaf warbler & & & $\mathrm{x}$ & $\mathrm{x}$ & $\mathrm{x}$ \\
\hline Rhipidura javanica & Pied fantail & & & $\mathrm{x}$ & $\mathrm{x}$ & $\mathrm{x}$ \\
\hline Zosterops montanus & Mountain white eye & & & $\mathrm{x}$ & $\mathrm{x}$ & $\mathrm{x}$ \\
\hline Ixobrychus cinnamomeus & Cinnamon bittern & $\mathrm{x}$ & & & & $\mathrm{x}$ \\
\hline Tanygnathus lucionensis & Blue naped parrot & $\mathrm{x}$ & & & & $\mathrm{x}$ \\
\hline $\begin{array}{l}\text { Spilornis cheela } \\
\text { Pachycephala }\end{array}$ & Crested serpent eagle & $\mathrm{x}$ & & $\mathrm{x}$ & & \\
\hline philippinensis & Yellow bellied whistler & & $\mathrm{x}$ & $\mathrm{x}$ & & \\
\hline Dicaem anthonyi & Flame crowned flower pecker & & $\mathrm{x}$ & & $\mathrm{x}$ & \\
\hline Egretta garzetta & Little egret & & $\mathrm{x}$ & & $\mathrm{x}$ & \\
\hline Eudynamys scolopacea & Common koel & & $\mathrm{x}$ & & & $\mathrm{x}$ \\
\hline Orthotomus castaneiceps & Philippine tailorbird & & $\mathrm{x}$ & & & $\mathrm{x}$ \\
\hline Motacilla alba & White wagtail & & & & $\mathrm{x}$ & $\mathrm{x}$ \\
\hline Aplonis panayensis & Asian glossy starling & & & $\mathrm{x}$ & & $\mathrm{x}$ \\
\hline Cisticola juncidis & Zitting cisticola & & & $\mathrm{x}$ & & $\mathrm{x}$ \\
\hline Ducula aenea & Green imperial pigeon & & & $\mathrm{x}$ & & $\mathrm{x}$ \\
\hline Eurylaimus steerii & Wattled broadbill & & & $\mathrm{x}$ & & $\mathrm{x}$ \\
\hline Micromacronous leytensis & Miniature tit babbler & & & $\mathrm{x}$ & & $\mathrm{x}$ \\
\hline Megapodius cumingii & Tabon scrubfowl & & & $\mathrm{x}$ & & $\mathrm{x}$ \\
\hline Passer mantanus & Eurasian tree sparrow & & & $\mathrm{x}$ & & $\mathrm{x}$ \\
\hline Pitta kochi & Whiskered pitta & & & $\mathrm{x}$ & & $\mathrm{x}$ \\
\hline Caprimulgus manillensis & Philippine nightjar & $x$ & & & & \\
\hline Macronous striaticeps & Brown tit babbler & $\mathrm{x}$ & & & & \\
\hline Porzana cinerea & White browed crake & $\mathrm{x}$ & & & & \\
\hline Anthus novaeseelandiae & Richard's pipit & & $\mathrm{x}$ & & & \\
\hline Lonchura leucogastra & White bellied munia & & $\mathrm{x}$ & & & \\
\hline Aethopyga pulcherrina & Metallic-winged sunbird & & & $\mathrm{x}$ & & \\
\hline Brachypteryx montana & White browed shortwing & & & $\mathrm{x}$ & & \\
\hline Chardrius leschenaultii & Greater sand-plover & & & $\mathrm{x}$ & & \\
\hline Lalage nigra & Pied triller & & & $\mathrm{x}$ & & \\
\hline Megalurus timoriensis & Tawny grassbird & & & $\mathrm{x}$ & & \\
\hline Pyrrbula leucogenis & White-cheeked bullfinch & & & $\mathrm{x}$ & & \\
\hline Rhipidura superciliaris & Blue fantail & & & $\mathrm{x}$ & & \\
\hline Turnix suscitator & Barred button quail & & & $\mathrm{x}$ & & \\
\hline Cacomantis variolosus & Brush cuckoo & & & & & $\mathrm{x}$ \\
\hline Ceyx lepidus & Variable dwarf-kingfisher & & & & & $\mathrm{x}$ \\
\hline Coturnix cbinensis & Blue breasted quail & & & & & $\mathrm{x}$ \\
\hline Dendrocygna arcuata & Wandering whistling duck & & & & & $\mathrm{x}$ \\
\hline Dicaeum trogonostigma & Orange-bellied flowerpecker & & & & & $\mathrm{x}$ \\
\hline Eumyias panayensis & Mountain verideter flycatcher & & & & & $\mathrm{x}$ \\
\hline Halcyon coromanda & Rudy kingfisher & & & & & $\mathrm{x}$ \\
\hline Megalurus palustris & Striated grassbird & & & & & $\mathrm{x}$ \\
\hline Nectarinia sperata & Purple throated sunbird & & & & & $\mathrm{x}$ \\
\hline $\begin{array}{l}\text { Padda oryzivora } \\
\text { Phaenicphaeus }\end{array}$ & Java sparrow & & & & & $\mathrm{x}$ \\
\hline curvirostris & Chestnut -breasted malkoha & & & & & $\mathrm{x}$ \\
\hline Ptilinopus lechancher & Black chinned fruit dove & & & & & $\mathrm{x}$ \\
\hline Tyto capensis & Grass owl & & & & & $\mathrm{x}$ \\
\hline
\end{tabular}


Table 2. Continuation

\begin{tabular}{|c|c|c|c|c|c|c|}
\hline Scientific Name & Common Name & $\underset{\stackrel{C}{\rightleftarrows}}{\stackrel{ \pm}{H}}$ & 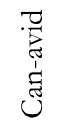 & 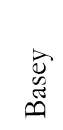 & 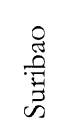 & 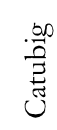 \\
\hline \multirow[t]{3}{*}{ Zoothera dauma } & Scaly ground thrush & & & & & $\mathrm{x}$ \\
\hline & Total number of species & 66 & 62 & 85 & 55 & 101 \\
\hline & Index of diversity & 3.25 & 3.66 & 3.71 & 3.48 & 3.92 \\
\hline
\end{tabular}

Among the 121 species of birds, 30 species were widely distributed 5 sites and are considered common; 21 species were present in 4 sites and 26 were present in 3 sites.

Twenty-seven species were recorded only in a single site, while 17 were observed only in 2 sites. Of the 27 species, which appeared to be in their restricted range as they were recorded only in a single site, 3 were recorded only in Taft (Caprimulgus manillensis, Macronous striaticeps and Porzana cinerea); 2 in Can-avid only (Anthus novaeseelandiae and Lonchura leucogastra); and 8 in Basey only (Aethopyga pulcherrina, Brachypteryx Montana, Chardrius leschenaultia, Lalage nigra, Megalurus timoriensis, Pyrrhula leucogenis, Rhipidura superciliaris and Turnix suscitator).

The highest number of restricted species of birds was obtained in Catubig with 14 species. These include Cacomantis variolosus, Ceyx Lepidus, Coturnix chinensis, Dendrocygna arcuata, Dicaeum trogonostigma, Eumyias panayensis, Halcyon coromanda, Megalurus palustris, Nectarinia sperata, Padda oryzivora, Phaenicphaeus curvirostris, Ptilinopus lechancher, Tyto capensis and Zoothera dauma.

Similarity patterns of bird species in the 5 priority watersheds were computed using the Jaccard index of similarity. The result showed high degree of similarity between watersheds which ranged from 0.43 to 0.63 (Table 3), which means that at least $43 \%$ of the bird species are shared between paired sites. Basey and Catubig had the highest value while Catubig and Taft had the lowest percentage. The result suggests that even if many species of birds are uniquely associated to particular watershed, many species are shared between the 5 five watersheds thus, similarity indices remain high. This condition does not call for highly site specific conservation measures when dealing with bird species within SINP.

Table 3. Similarity indices for birds recorded from five priority watersheds in Samar Island Natural Park, c. 2011

\begin{tabular}{lccccc}
\hline & Taft & Can-avid & Basey & Suribao & Catubig \\
\hline Taft & & 44 & 51 & 38 & 50 \\
Can-avid & 68.75 & & 48 & 44 & 54 \\
Basey & 67.54 & 65.31 & & 46 & 72 \\
Suribao & 62.81 & 75.21 & 65.71 & & 51 \\
Catubig & 59.88 & 66.26 & 77.42 & 65.38 & \\
\hline
\end{tabular}

*Values in shaded cells refer to the number of species common to both sites. 
MAMMALS

Species Composition

A total of 20 terrestrial mammals were recorded in the 5 priority watersheds (Table 4). These belonged to 7 orders and 12 families. Of these 20 mammals, nine (45\%) were endemic to the Philippines, four $(20 \%)$ of which were endemic to the greater Mindanao faunal region. The rest were resident species. The Mindanao endemics were Cynocephalus volans, Tarsius syrichta, Sundasciurus philipinensis and Nannosciurus surrutilus. These were observed in nearly all the sites and thus represent important biological indicators for Samar's lowland forests as suggested by the previous BRA.

Table 4. List of terrestrial mammals recorded from five priority watersheds in Samar Island Natural Park, c. 2011

\begin{tabular}{|c|c|c|c|c|}
\hline Order & Family & Scientific Name & Common Name & Status* \\
\hline \multirow[t]{2}{*}{ Artiodactyla } & Cervidae & Cervus mariannus & Philippine Brown Deer & Philippine Endemic \\
\hline & Suidae & Sus philippensis & Philippine Warty Pig & Philippine Endemic \\
\hline \multirow[t]{2}{*}{ Carnivora } & Viveridae & Paradoxurus hermaphroditus & Common Palm Civet & Resident \\
\hline & & Vivera tagalunga & Malay Civet & Resident \\
\hline \multirow[t]{7}{*}{ Chiroptera } & Pteropodidae & Acerodon jubatus & Golden-crowned Flying Fox & Philippine Endemic \\
\hline & & Cynopterus brachyotis & Short-nosed Fruit Bat & Resident \\
\hline & & Rousettus amplexicaudatus & Common Rousette & Resident \\
\hline & & Pteropus vampyrus & Large Flying Fox & Resident \\
\hline & Rhinolophidae & Rhinolophus inops & Philippine Forest & Philippine Endemic \\
\hline & & & Horseshoe Bat & \\
\hline & Vespertilionidae & Scotophilus kublii & Lesser Asian House Bat & Resident \\
\hline Dermoptera & Cynocephalidae & Cynocephalus volans & Philippine Flying Lemur & Mindanao Endemic \\
\hline Insectivora & Soricidae & Suncus marinus & Asian House Shrew & Resident \\
\hline \multirow[t]{2}{*}{ Primates } & Cercopithecidae & Macaca fascicularis & Long-tailed Macaque & Resident \\
\hline & Tarsiidae & Tarsius syrichta & Philippine Tarsier & Mindanao Endemic \\
\hline \multirow[t]{6}{*}{ Rodentia } & Muridae & Rattus everetti & $\begin{array}{l}\text { Common Philippine Forest } \\
\text { Rat }\end{array}$ & Philippine Endemic \\
\hline & & Rattus exulans & Polynesian Rat & Resident \\
\hline & & Rattus mindanensis & Common Field Rat & Resident \\
\hline & & Rattus tane zumi & Oriental House Rat & Resident \\
\hline & Scuiridae & Sundasciurus pbilipinensis & Philippine Tree Squirrel & Mindanao Endemic \\
\hline & & Nannosciurus surrutilus & Mindanao Pygmy Squirrel & Mindanao Endemic \\
\hline
\end{tabular}

Source: Rabor, 1986

Seven species of mammals were considered threatened under DENR DAO 2004-15. These include the endangered Acerodon jubalus and the vulnerable Cervus mariannus and Sus philippensis. Other threatened species include the Cynocephalus volans, Tarsius syrichta, Macaca fascicularis and Pteropus vampyrus.

It is quite interesting to note that majority (71\%) of the threatened species of terrestrial mammals recorded in the present study are endemic to the Philippines with 2 (28\%) endemic to the greater Mindanao faunal region. Most of these are forest dependents and are threatened due to habitat loss and hunting. Although some can tolerate a certain degree of disturbance they still prefer undisturbed forests as habitat. 


\section{Mammalian Species Distribution and Similarity}

The distribution of mammals in the five priority watersheds is shown in Table 5. Highest number of species of 17 was observed in Catubig followed by Taft with 16. Basey had the lowest number of 9 species. Four species of mammals (Sundasciurus philipinensis, Acerodon jubatus, Scotophilus kuhlii and Nannosciurus surrutilus) were considered fairly common being present only in 3 sites. Another four species (Rousettus amplexicaudatus, Rattus everetti, Cynocephalus volans and Rattus mindanensis) had limited distribution in 2 sites.

Table 5. Distribution of terrestrial mammals recorded from five priority watersheds in Samar Island Natural Park, c. 2011

\begin{tabular}{|c|c|c|c|c|c|c|}
\hline Scientific Name & Common Name & 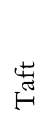 & 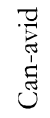 & 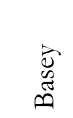 & 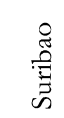 & $\frac{\frac{a 0}{3}}{\frac{E}{E}}$ \\
\hline Cervus mariannus & Philippine Brown Deer & $\mathrm{x}$ & $\mathrm{x}$ & $x$ & $\mathrm{x}$ & $\mathrm{x}$ \\
\hline Sus philippensis & Philippine Warty Pig** & $\mathrm{x}$ & $\mathrm{x}$ & $\mathrm{x}$ & $\mathrm{x}$ & $\mathrm{x}$ \\
\hline Paradoxurus & Common Palm Civet & $\mathrm{x}$ & $\mathrm{x}$ & $\mathrm{x}$ & $\mathrm{x}$ & $\mathrm{x}$ \\
\hline \multicolumn{7}{|l|}{ hermaphroditus } \\
\hline Vivera tagalunga & Malay Civet & $x$ & $\mathrm{x}$ & $x$ & $\mathrm{x}$ & $\mathrm{x}$ \\
\hline Pteropus vampyrus & Large Flying Fox* & $\mathrm{x}$ & $\mathrm{x}$ & $\mathrm{x}$ & $\mathrm{x}$ & $\mathrm{x}$ \\
\hline Suncus marinus & Asian House Shrew & $\mathrm{x}$ & $\mathrm{x}$ & $\mathrm{x}$ & $\mathrm{x}$ & $\mathrm{x}$ \\
\hline Macaca fascicularis & Long-tailed Macaque* & $\mathrm{x}$ & $\mathrm{x}$ & $\mathrm{x}$ & $\mathrm{x}$ & $\mathrm{x}$ \\
\hline Rattus tanezumi & Oriental House Rat & $\mathrm{x}$ & $\mathrm{x}$ & $\mathrm{x}$ & $\mathrm{x}$ & $\mathrm{x}$ \\
\hline Tarsius syrichta & Philippine Tarsier* & $\mathrm{x}$ & & $\mathrm{x}$ & $\mathrm{x}$ & $\mathrm{x}$ \\
\hline Sundasciurus philipinensis & Philippine Tree Squirrel & $\mathrm{x}$ & $\mathrm{x}$ & & $\mathrm{x}$ & \\
\hline Acerodon jubatus & Golden-crowned Flying Fox & $\mathrm{x}$ & $\mathrm{x}$ & & & $\mathrm{x}$ \\
\hline Scotophilus kublii & Lesser Asian House Bat & $\mathrm{x}$ & $\mathrm{x}$ & & & $\mathrm{x}$ \\
\hline Nannosciurus surrutilus & Mindanao Pygmy Squirrel & & $\mathrm{x}$ & & $\mathrm{x}$ & $\mathrm{x}$ \\
\hline Rousettus amplexicaudatus & Common Rousette & $\mathrm{x}$ & & & & $\mathrm{x}$ \\
\hline Rattus everetti & Common Philippine Forest Rat & $\mathrm{x}$ & & & & $\mathrm{x}$ \\
\hline Cynocephalus volans & Philippine Flying Lemur** & & $\mathrm{x}$ & & & $\mathrm{x}$ \\
\hline Rattus mindanensis & Common Field Rat & & & & $\mathrm{x}$ & $\mathrm{x}$ \\
\hline Cynopterus brachyotis & Short-nosed Fruit Bat & $\mathrm{x}$ & & & & \\
\hline Rattus exulans & Polynesian Rat & $\mathrm{x}$ & & & & \\
\hline \multirow{2}{*}{ Rhinolophus inops } & Philippine Forest Horseshoe Bat & & & & & $\mathrm{x}$ \\
\hline & & 16 & 13 & 9 & 12 & 17 \\
\hline
\end{tabular}

Very few species of mammals showed very limited distribution having been recorded only in a single site. Cynopterus brachyotis and Rattus exulans were recorded only in Taft watershed while Rhinolophus inops was observed only in Catubig. Two species of rats (Rattus mindanensis and Rattus exulans) which were commensal species were recorded in some sites. Their presence may somehow indicate some degree of habitat degradation in these watersheds due to expansion of clearings for cultivation and settlements.

The result showed high degree of similarity between watersheds which ranged from 0.53 to 0.75 (Table 6). This means that approximately at least $53 \%$ of the species were present in paired sites. The highest value was observed between Basey and Suribao while the lowest value was observed 
between Basey and Catubig. Identical similarity index was observed between Can-avid compared to Suribao and Catubig. The result suggest that many species of mammals were shared between the 5 watersheds as evidenced by close similarity values. This may somehow show that the 5 watersheds exhibit similar habitat conditions which means similar management intervention when addressing mammalian species.

Table 6. Similarity indices for terrestrial mammals recorded from five priority watersheds in Samar Island Natural Park, c. 2011

\begin{tabular}{lccccc}
\hline & Taft & Can-avid & Basey & Suribao & Catubig \\
\hline Taft & & 11 & 9 & 10 & 13 \\
Can-avid & 75.86 & & 8 & 10 & 12 \\
Basey & 72.00 & 72.73 & & 9 & 9 \\
Suribao & 71.43 & 80.00 & 85.71 & & 11 \\
Catubig & 78.79 & 80.00 & 69.23 & 75.86 & \\
\hline
\end{tabular}

*Values in shaded cells refer to the number of species common to both sites.

\section{REPTILES}

\section{Species Composition}

A total of 23 reptiles belonging to 4 orders and 9 families were recorded in the 5 priority watersheds of SINP (Table 7). Of these, ten (43\%) were endemic to the Philippines of which three $(13 \%)$ were endemic to the greater Mindanao faunal region. The most represented family was Scincidae with 10 species of ground and tree dwelling skinks which were mostly common in secondary and primary forest. Colubridae was next most represented family with 3 species of snakes. Geckonidae, Agamidae and Elapidae 2 species each. The two species of geckos (Gekko gecko and Lepidodactylus planicaudus) are associated to human dwellings. Agamidae was represented by two endemic species of lizards (Draco mindanensis and Hydrosaurus pustulatus) while Elapidae with 2 species of cobras (Naja samarensis and Ophiophagus hannah). The Samar Cobra (Naja samarensis) is a subspecies of the Philippine Cobra endemic to the place and the greater Mindanao faunal region (Alcala, 1986).

Two species of reptiles were considered threatened under DENR DAO 2004-15 and Vulnerable by IUCN (IUCN, 2015). These included the Hydrosaurus pustulatus and Python reticulatus. The presence of the critically endangered Philippine crocodile (Crocodylus mindorensis) in the 5 priority watersheds which was reported in the previous BRA cannot be confirmed by local informants.

Although relatively low for Samar, the present record of reptiles can still demonstrate interesting habits and specialized feeding adaptations which is reflective of the overall health of Samar lowland forest ecosystem. 
Table 7. List of reptiles recorded from five priority watersheds in Samar Island Natural Park, c. 2011

\begin{tabular}{|c|c|c|c|c|}
\hline Order & Family & Scientific Name & Common Name & Status* \\
\hline \multirow[t]{12}{*}{ Squamata } & Scincidae & Brachymeles gracilis & Common Burrowing Skink & Philippine Endemic \\
\hline & & Lipinia pulchellum. & $\begin{array}{l}\text { Yellow-striped Slender } \\
\text { Tree skink }\end{array}$ & Philippine Endemic \\
\hline & & Lamprolepis smaragdina & Spotted Green Tree Skink & Resident \\
\hline & & Mabuya multicarinata & Two-striped Mabouya & Resident \\
\hline & & Mabuya multifasciata & Common Mabouya & Resident \\
\hline & & Otosaurus cummingi & Cumming's Eared Skink & Philippine Endemic \\
\hline & & Sphenomorphus acutus & $\begin{array}{l}\text { Point-headed } \\
\text { Sphenomorphus }\end{array}$ & Philippine Endemic \\
\hline & & Sphenomorphus coxi & Cox's Sphernomorphus & Philippine Endemic \\
\hline & & Sphenomorphus fasciatus & Banded Sphernomorphus & Philippine Endemic \\
\hline & & Tropidophorus grayi & Spiny Waterside Skink & Resident \\
\hline & Geckonidae & Gekeko gecko & Tokay Gecko & Resident \\
\hline & & $\begin{array}{l}\text { Lepidodactylus } \\
\text { planicaudus }\end{array}$ & Smooth-scaled gecko & Resident \\
\hline \multirow[t]{3}{*}{ Sauria } & Varanidae & Varanus salvator & Gray Monitor Lizard & Resident \\
\hline & Agamidae & Draco mindanensis & Mindanao Flying Lizard & Mindanao Endemic \\
\hline & & Hydrosaurus pustulatus & Philippine Sailfin Lizard & Philippine Endemic \\
\hline \multirow[t]{7}{*}{ Serpentes } & Boidae & Python reticulatus & Reticulated Python & Resident \\
\hline & Elapidae & Naja samarensis & Samar Cobra & Mindanao Endemic \\
\hline & & Ophiophagus hannah & King Cobra & Resident \\
\hline & Colubridae & Calamaria lumbricoidea & Dark-bellied Worm Snake & Resident \\
\hline & & Dendrelaphis puctualata & $\begin{array}{l}\text { Common Green Tree } \\
\text { Snake }\end{array}$ & Resident \\
\hline & & $\begin{array}{l}\text { Macropophis } \\
\text { dendrophiops }\end{array}$ & Spotted Water Snake & Mindanao Endemic \\
\hline & Viperidae & Tropidolaemus wagleri & Wagler's Pit-viper & Resident \\
\hline Testudinata & Emydidae & Cuora amboinensis & Malayan Freshwater Turtle & Resident \\
\hline
\end{tabular}

Source: Alcala, 1986

\section{Reptile Species Distribution and Similarity}

The distribution of reptiles in the five priority watersheds is shown in Table 8. Highest number of species of 17 was observed in Basey while the lowest was in Can-avid with 12. Eight species (Cuora amboinensis, Gekko gecko, Lepidodactylus planicaudus, Hydrosaurus pustulatus, Naja samarensis, Ophiophagus Hannah, and Python reticulatus) were recorded in all 5 sites. Only one species was observed in 4 sites.

Five species had limited distribution to only 1 site. Sphenomorphus acutus, Calamaria lumbricoidea, and Dendrelaphis puctualata were recorded only in Basey watershed while Sphenomorphus fasciatus and Sphenomorphus coxi were observed only in Suribao and Catubig, respectively.

The similarity indices of reptiles for the 5 priority watersheds are shown in Table 9. The result showed high degree of similarity between watersheds which ranged from 0.42 to 0.73 . Highest value was observed identical between Taft and Suribao. Lowest value was observed between Taft and Catubig, Can-avid and Suribao. Two paired sites (Taft versus Canavid and Suribao versus Can-avid) have identical index of 0.67. The result suggests that many species of reptiles were shared between the 5 watersheds as evidenced by close similarity values thus, does not call for highly site specific conservation measures. 
Table 8. Distribution of reptiles recorded from five priority watersheds in Samar Island Natural Park, c. 2011

\begin{tabular}{|c|c|c|c|c|c|c|}
\hline Scientific Name & Common Name & $\underset{ت}{\stackrel{\leftrightarrows}{\leftrightarrows}}$ & 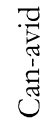 & 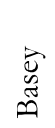 & 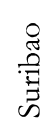 & $\frac{\cdot \frac{b}{B}}{\stackrel{\vec{E}}{\Xi}}$ \\
\hline Cuora amboinensis & Malayan Freshwater Turtle & $\mathrm{x}$ & $\mathrm{x}$ & $\mathrm{x}$ & $\mathrm{x}$ & $\mathrm{x}$ \\
\hline Gekeko gecko & Tokay Gecko & $\mathrm{x}$ & $\mathrm{x}$ & $\mathrm{x}$ & $\mathrm{x}$ & $\mathrm{x}$ \\
\hline Lepidodactylus planicaudus & Smooth-scaled gecko & $\mathrm{x}$ & $\mathrm{x}$ & $\mathrm{x}$ & $\mathrm{x}$ & $\mathrm{x}$ \\
\hline Hydrosaurus pustulatus & Philippine Sailfin Lizard & $\mathrm{x}$ & $\mathrm{x}$ & $\mathrm{x}$ & $\mathrm{x}$ & $\mathrm{x}$ \\
\hline Naja samarensis & Samar Cobra* & $\mathrm{x}$ & $\mathrm{x}$ & $\mathrm{x}$ & $\mathrm{x}$ & $\mathrm{x}$ \\
\hline Ophiophagus hannah & King Cobra & $\mathrm{x}$ & $\mathrm{x}$ & $\mathrm{x}$ & $\mathrm{x}$ & $\mathrm{x}$ \\
\hline Python reticulatus & Reticulated Python* & $\mathrm{x}$ & $\mathrm{x}$ & $\mathrm{x}$ & $\mathrm{x}$ & $\mathrm{x}$ \\
\hline Varanus salvator & Gray Monitor Lizard* & $\mathrm{x}$ & $\mathrm{x}$ & $\mathrm{x}$ & $\mathrm{x}$ & $\mathrm{x}$ \\
\hline Brachymeles gracilis & Common Burrowing Skink & $\mathrm{x}$ & $\mathrm{x}$ & $\mathrm{x}$ & $\mathrm{x}$ & \\
\hline Mabuya multifasciata & Common Mabouya & $\mathrm{x}$ & $\mathrm{x}$ & & $\mathrm{x}$ & \\
\hline Draco mindanensis & Mindanao Flying Lizard & & $\mathrm{x}$ & $\mathrm{x}$ & & $\mathrm{x}$ \\
\hline Tropidophorus grayi & Spiny Waterside Skink & $\mathrm{x}$ & & $\mathrm{x}$ & & \\
\hline Tropidolaemus wagleri & Wagler's Pit-viper & $\mathrm{x}$ & & $\mathrm{x}$ & & \\
\hline Lamprolepis smaragdina & Spotted Green Tree Skink & $\mathrm{x}$ & & & $\mathrm{x}$ & \\
\hline Lipinia pulchellum. & Yellow-striped Slender Tree skink & & $\mathrm{x}$ & & & $\mathrm{x}$ \\
\hline Otosaurus cummingi & Cumming's Eared Skink & & & $\mathrm{x}$ & & $\mathrm{x}$ \\
\hline Macropophis dendrophiops & Spotted Water Snake & & & $\mathrm{x}$ & & $\mathrm{x}$ \\
\hline Mabuya multicarinata & Two-striped Mabouya & & & & $\mathrm{x}$ & $\mathrm{x}$ \\
\hline Sphenomorphus acutus & Point-headed Sphenomorphus & & & $\mathrm{x}$ & & \\
\hline Calamaria lumbricoidea & Dark-bellied Worm Snake & & & $\mathrm{x}$ & & \\
\hline Dendrelaphis puctualata & Common Green Tree Snake & & & $\mathrm{x}$ & & \\
\hline Sphenomorphus fasciatus & Banded Sphernomorphus & & & & $\mathrm{x}$ & \\
\hline \multirow[t]{2}{*}{ Sphenomorphus coxi } & Cox's Sphernomorphus & & & & & $\mathrm{x}$ \\
\hline & & 13 & 12 & 17 & 13 & 14 \\
\hline
\end{tabular}

Table 9. Similarity indices for reptiles recorded from five priority watersheds in Samar Island Natural Park, c. 2011

\begin{tabular}{lccccc}
\hline & Taft & Can-avid & Basey & Suribao & Catubig \\
\hline Taft & & 10 & 11 & 11 & 8 \\
Can-avid & 80.00 & & 10 & 10 & 10 \\
Basey & 73.33 & 68.97 & & 9 & 11 \\
Suribao & 84.62 & 80.00 & 60.00 & & 9 \\
Catubig & 59.26 & 76.92 & 70.97 & 66.67 & \\
\hline
\end{tabular}

*Values in shaded cells refer to the number of species common to both sites.

\section{AMPHIBIANS}

\section{Species Composition}

A total of 18 species of amphibians belonging to 4 families were recorded from the 5 priority watersheds (Table 10). Of the 18 species, two were introduced (Bufo marinus and Rana erythraea) and the rest were native. Seven (39\%) species of amphibians were endemic to the Philippines. Only one species of amphibian was considered vulnerable under DENR DAO 2004-15 and IUCN. This is the Platymantis rabori. 
This Mindanao endemic species inhabits arboreal microhabitats in lower montane and lowland forests. It breeds by direct development and lays its eggs in nests in tree ferns and pandans. The major threat is the loss of lowland rainforest due to small-scale logging, conversion to agricultural land and mining (IUCN, 2015).

Table 10. List of amphibians recorded from five priority watersheds in Samar Island Natural Park, c. 2011

\begin{tabular}{cllll}
\hline Order & Family & Scientific Name & Common Name & Status* \\
\hline Anura & Bufonidae & Bufo marinus & Giant Marine toad & Introduced \\
& Microhylidae & Kalophrynus pleurostigma & Spotted Narrow-mouthed & Resident \\
& Pelobatidae & Megophrys monticola & Horned litter toad & Resident \\
Ranidae & Limnonectes magna & Giant Philippine frog & Endemic \\
& Occidozyga laevis & Common puddle frog & Resident \\
& Platimantis dorsalis & Common Forest Frog & Endemic \\
& Platimantis rabori & Rabor Forest Frog & Endemic \\
& Rana erythraea & Common Green Frog & Resident \\
& Rana everitti & Everett's frog & Endemic \\
& Rana limnocharis & Common Ricefield Frog & Resident \\
& Rana microdisca & Small-disk Frog & Resident \\
& Rana signata & Variable-backed Frog & Resident \\
& Rana woodworthi & Woodsworth's frog & Endemic \\
& Staurois natator & Rock frog & Resident \\
& Philautus leitensis & Leyte forest tree frog & Endemic \\
& Philautus surdus & Common Forest Tree Frog & Endemic \\
& Polypedates leucomystax & Common Tree Frog & Resident \\
& Rhacophorus bimaculatus & Asian Tree Frog & Resident \\
\hline
\end{tabular}

Source: Alcala, 1986

One species of frog found in Samar is of special concern due to its apparently limited distribution.The island endemic Samar tree frog (Polypedates hecticus) is known only in Samar island, and found nowhere else in the world. This species is however listed as Data Deficient by IUCN (IUCN, 2015) in view of the absence of recent information on its extent of occurrence, status and ecological requirements. Unfortunately, the present study was not able to record this unique anuran species. As pointed out in the 2003 study, the Samar Tree Frog along with other island-endemic species increases the value of the island's remaining forest habitats as a vital refuge for many other restricted-range taxa, putting higher global priority into these watersheds for biodiversity conservation.

The relatively low number of amphibians recorded in the present study as compared to the 2003 BRA does not mean decline of amphibians in SINP and should not be considered alarming because the presence of indicator species which demonstrate that the environmental health of SINP is still good. As in the previous BRA, resource partitioning among frogs in the multi-layer lowland evergreen rainforest of Samar Island, has been very evident by representation of certain species to occupy distinct microhabitats within the forest - canopy layer, understory, undergrowth and forest floor, each with unique adaptations for survival. Based on the 
present survey, amphibians found inhabiting forest floor include Kalophrynus pleurostigma and Megophrys monticola; amphibians inhabiting the moist undergrowth include Philautus leitensis and Philautus surdus; amphibians found in riverine habitats include Limnonectes magna, Staurois natator, Occidozyga laevis and Rana signat; arboreal amphibians inhabiting forest canopy include Rhacophorus pardalis) and Polypedates leucomystax.

\section{Amphibian Species Distribution and Diversity}

The distribution of amphibians in the five priority watersheds is shown in Table 11. Highest number of species of 14 was observed in Taft followed by Catubig with 13. Can-avid, Basey and Suribao had equal number of 9 species. Four species of ranids (Occidozyga laevis, Rana everitti, Rana woodworthi, Philautus leitensis) were recorded in all sites. Five species (Staurois natator, Platimantis dorsalis, Rana signata, Limnonectes magna, Megophrys monticola) were observed to be common 4 sites. Five species had limited distribution to 2 sites. These include Bufo marinus, Rana erythraea, Rhacophorus bimaculatus, Kalophrynus pleurostigma, and Polypedates leucomystax. Four species had very limited distribution and were observed only in a single site. These include Platimantis rabori and Rana limnocharis which were observed only in Taft and Rana microdisca and Philautus surdus which were recorded only in Catubig.

Table 11. Distribution of amphibians recorded from five priority watersheds in Samar Island Natural Park, c. 2011

\begin{tabular}{|c|c|c|c|c|c|c|}
\hline Scientific Name & Common Name & $\underset{H}{\stackrel{\varpi}{\rightleftarrows}}$ & 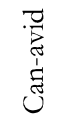 & $\begin{array}{l}\hat{y} \\
\tilde{\widetilde{J}} \\
\oplus\end{array}$ & 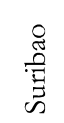 & 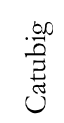 \\
\hline Occidozyga laevis & Common puddle frog & $\mathrm{x}$ & $\mathrm{x}$ & $\mathrm{x}$ & $\mathrm{x}$ & $\mathrm{x}$ \\
\hline Rana everitti & Everett's frog & $\mathrm{x}$ & $\mathrm{x}$ & $\mathrm{x}$ & $\mathrm{x}$ & $\mathrm{x}$ \\
\hline Rana woodworthi & Woodsworth's frog & $\mathrm{x}$ & $\mathrm{x}$ & $\mathrm{x}$ & $\mathrm{x}$ & $\mathrm{x}$ \\
\hline Philautus leitensis & Leyte forest tree frog & $\mathrm{x}$ & $\mathrm{x}$ & $\mathrm{x}$ & $\mathrm{x}$ & $\mathrm{x}$ \\
\hline Staurois natator & Rock frog & $\mathrm{x}$ & $\mathrm{x}$ & $\mathrm{x}$ & $\mathrm{x}$ & \\
\hline Platimantis dorsalis & Common Forest Frog & $\mathrm{x}$ & $\mathrm{x}$ & $\mathrm{x}$ & & $\mathrm{x}$ \\
\hline Rana signata & Variable-backed Frog & $\mathrm{x}$ & $\mathrm{x}$ & & $\mathrm{x}$ & $\mathrm{x}$ \\
\hline Limnonectes magna & Giant Philippine frog & $\mathrm{x}$ & $\mathrm{x}$ & & $\mathrm{x}$ & $\mathrm{x}$ \\
\hline Megophrys monticola & Horned litter toad & $\mathrm{x}$ & & $\mathrm{x}$ & $\mathrm{x}$ & $\mathrm{x}$ \\
\hline Bufo marinus & Giant Marine toad & $\mathrm{x}$ & $\mathrm{x}$ & & & \\
\hline Rana erythraea & Common Green Frog & $\mathrm{x}$ & & & $\mathrm{x}$ & \\
\hline Rhacophorus bimaculatus & Asian Tree Frog & $\mathrm{x}$ & & & & $\mathrm{x}$ \\
\hline Kalophrymus pleurostigma & Spotted Narrow-mouthed frog & & & $\mathrm{x}$ & & $\mathrm{x}$ \\
\hline Polypedates leucomystax & Common Tree Frog & & & $\mathrm{x}$ & & $\mathrm{x}$ \\
\hline Platimantis rabori & Rabor Forest Frog & $\mathrm{x}$ & & & & \\
\hline Rana limnocharis & Common Ricefield Frog & $\mathrm{x}$ & & & & \\
\hline Rana microdisca & Small-disk Frog & & & & & $\mathrm{x}$ \\
\hline Philautus surdus & Common Forest Tree Frog & & & & & $\mathrm{x}$ \\
\hline & Total Number of Species & 14 & 9 & 9 & 9 & 13 \\
\hline & Index of Diversity & 2.13 & 2.01 & 1.83 & 1.93 & 1.97 \\
\hline
\end{tabular}


The indices of diversity of amphibians for the five priority watersheds were fairly high with values ranging from 1.83 to 2.13 . The highest index was observed in Taft watershed while the lowest in Basey watershed. The fairly high indices could be due to the high degree of abundance in amphibians along stream transects in which observations were made. Observations outside transect were not included in the computation.

The presence of Bufo marinus in Taft and Can-avid and Rana erythraea in Taft and Suribao may somehow indicate some degree of habitat degradation in these watersheds due to expansion of clearings for cultivation and settlements.

\section{Amphibian Species Similarity}

Similarity pattern of amphibians in the 5 priority watersheds was computed using the Sorensen's index of similarity. The similarity indices of amphibians for the 5 priority watersheds are shown in Table 12. The result showed high degree of similarity between watersheds which ranged from 0.61 to 0.78 . which means that more than $60 \%$ of the species were present in 2 sites. Highest value was observed identical between Taft compared to Basey and Suribao. Lowest value was observed identical when Catubig was compared to Can-avid and Suribao. The result suggest that many species of amphibians were shared between the 5 watersheds as evidenced by close similarity values thus, does not call for highly site specific conservation measures.

Table 12. Similarity indices for amphibians recorded from five priority watersheds in Samar Island Natural Park, c. 2011

\begin{tabular}{lccccc}
\hline & Taft & Can-avid & Basey & Suribao & Catubig \\
\hline Taft & & 9 & 7 & 9 & 9 \\
Can-avid & 0.64 & & 6 & 7 & 7 \\
Basey & 0.43 & 0.50 & & 6 & 8 \\
Suribao & 0.64 & 0.63 & 0.50 & & 7 \\
Catubig & 0.50 & 0.46 & 0.57 & 0.46 & \\
\hline
\end{tabular}

*Values in shaded cells refer to the number of species common to both sites.

\section{CONSERVATION ISSUES}

The species richness of the lowland forest of Samar within the SINP in terms of terrestrial vertebrates is remarkably high. In addition to its high diversity, Samar contains a significant number of endemic faunal species whose contribution is limited to the greater Mindanao faunal region, most of which are recognized by both the national authority and international conservation groups as threatened. The presence of these species further stresses the need to make SINP a conservation priority site in the Philippines.

The most serious threat to terrestrial fauna in SINP is hunting and capture for both subsistence and for commercial trade. The primary target 
species are the big games particularly wild pig and deer because of their large amount of meat per catch and the associated high price if traded commercially. The population of deer however has declined tremendously in recent years according to local informants, so that most commercial hunters in the area focus only in the pursuit of wild pig. With the changing trend from subsistence to commercial hunting in wild pig, sophistication in hunting techniques has also improved to increase catch per hunting effort.

Habitat alteration is another threat to wildlife in SINP. Natural habitats, especially the primary and secondary forests are under increasing pressure from growing human populations and unsound resource utilization. The conversion of forest to agricultural land use and small-scale illegal logging operations or timber poaching is a serious threat. Aside from lumber, local residents are also extracting timber to be made into boat keels, which is indirectly linked to the fishing industry and local navigational transport system within the island. Clearing of forests for charcoal making which has become very rampant is also a significant threat.

Clearing of forests to some extent may be beneficial to wildlife because of increased habitat diversity and complexity due to increased horizontal stratification. However if the current rate of forest clearance continues, forests may be decimated to the disadvantage of those highly sensitive interior species of wildlife, which are mostly affected by the habitat transformation or fragmentation. Because fragmentation disrupts species ecology and reproduction, this means that populations of already threatened species can very quickly fall below minimum viable level so that their long-term prospect for survival becomes bleak.

The combination of high species richness, high regional endemism, and unique wildlife assemblages should make Samar forests a high conservation priority. It is highly recommended to protect what remains of these important lowland forests. Aside from protection, considering the on-going destruction and degradation of Samar forests within SINP brought about by economic struggle of local inhabitants, there is therefore an urgent need to implement some conservation measures. Such conservation measures must integrate economical viable and socially acceptable livelihood alternatives for the local communities in order to lessen their dependence on the resources of SINP.

\section{CONCLUSION AND RECOMMENDATION}

Results of the study showed that Samar holds high diversity, high endemism and an unusual combination of terrestrial vertebrate species. It contains a remarkable number of threatened species being recognized by the international arena in biological diversity conservation, most of which have strong affinity to the Mindanao faunal region. Although there is still a clear need to continue faunal assessment in Samar island, the existing data are sufficient to further highlight the need to establish SINP as 
conservation priority site in the region.

Hunting and habitat destruction must be put to complete stop to preserve what is left of the wildlife resources in the SINP. Along with strict enforcement of laws, effective information dissemination and relevant educational efforts could convince the local people to protect the remaining wildlife resources being an important heritage of the Samarenos.

\section{REFERENCES}

ALCALA, A.C. 1986. Guide to Philippine Flora and Fauna: Amphibians and Reptiles. University of the Philippines. 10:1-195.

ALCALA, A.C. and W.C. BROWN. 1998. Philippine Amphibians: an illustrated field guide. Bookmark Inc., Makati City, Philippines. 113p

DENR. 2004. Department Administrative Order 2004-15. Establishing the List of Terrestrial Threatened Species and their Categories, and the List of Other Wildlife Species Pursuant to Republic Act No. 9147, otherwise known as the Wildlife Resources Conservation Act and Protection of 2001.Quezon City: Department of Environment and Natural Resources.

GONZALES, P.C. and C. P. REES. 1988. Birds of the Philippines. Haribon Foundation for the Conservation of Natural Resources, Inc. Manila, Philippines. $184 \mathrm{p}$.

IUCN. 2015. The IUCN Red List of Threatened Species 2015: www.iucnredlist.org.

KENNEDY, R.S., P.C. GONZALES, E.H. DICKINSON, H.C. MIRANDA Jr., and T.H. FISHER. 2000. A guide to the Birds of the Philippines. Oxford University Press. New York. 369 p.

MAGURRAN, A.E. 1988. Ecological Diversity and Its Measurement. Princeton University Press. 179 p.

MALLARI, N.A.D, B.R. TABARANZA and M.J. CROSBY. 2001. Key Conservation Sites in the Philippines: a Haribon Foundation and Birdlife International Directory of Important Bird Areas. DENR and Bookmark, Inc. 485 p.

MALLARI, N.A.D. 1992. Species diversity along elevational gradients in different study sites in Sierra Madre Mountains in Isabela and Cagayan. B.S. Thesis, UP at Los Baños. 88 p.

RABOR, D.S. 1986. Guide to Philippine Flora and Fauna: Birds and Mammals. University of the Philippines. 2:1-284

SEAMEO-SEARCA. 2004. Biological resources assessment - Samar Island Natural Park, Philippines. PAWB, DENR-8, Tacloban City. 191p.

UNDP-GEF-DENR. 2007. Samar Island Natural Park Management Plan June 2006 -May 2016. DENR-R8, Tacloban City. 249p. 